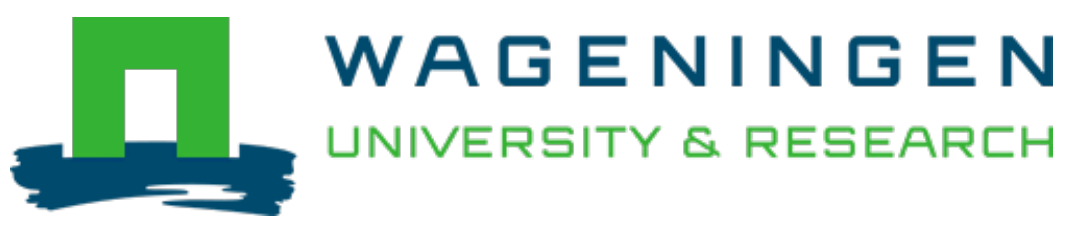

\title{
Hooked on mushrooms : Preparation and mechanics of a bioinspired soft probabilistic fastener
}

\author{
Biointerphases \\ Sharma, Preeti; Saggiomo, Vittorio; Doef, Vincent; Kamperman, Marleen; A. Dijksman, Joshua \\ https://doi.org/10.1116/6.0000634
}

This article is made publicly available in the institutional repository of Wageningen University and Research, under the terms of article $25 \mathrm{fa}$ of the Dutch Copyright Act, also known as the Amendment Taverne. This has been done with explicit consent by the author.

Article 25 fa states that the author of a short scientific work funded either wholly or partially by Dutch public funds is entitled to make that work publicly available for no consideration following a reasonable period of time after the work was first published, provided that clear reference is made to the source of the first publication of the work.

This publication is distributed under The Association of Universities in the Netherlands (VSNU) 'Article $25 \mathrm{fa}$ implementation' project. In this project research outputs of researchers employed by Dutch Universities that comply with the legal requirements of Article $25 \mathrm{fa}$ of the Dutch Copyright Act are distributed online and free of cost or other barriers in institutional repositories. Research outputs are distributed six months after their first online publication in the original published version and with proper attribution to the source of the original publication.

You are permitted to download and use the publication for personal purposes. All rights remain with the author(s) and / or copyright owner(s) of this work. Any use of the publication or parts of it other than authorised under article $25 \mathrm{fa}$ of the Dutch Copyright act is prohibited. Wageningen University \& Research and the author(s) of this publication shall not be held responsible or liable for any damages resulting from your (re)use of this publication.

For questions regarding the public availability of this article please contact openscience.library@,wur.nl 


\title{
Hooked on Mushrooms: Preparation and Mechanics of a Bioinspired Soft Probabilistic Fastener
}

\author{
Preeti Sharma*1,, Vittorio Saggiomo*2, Vincent van der Doef ${ }^{1}$, Marleen Kamperman ${ }^{3}$, and \\ Joshua A. Dijksman ${ }^{1, \dagger}$ \\ ${ }^{1}$ Physical Chemistry and Soft Matter, Wageningen University $\&$ Research, Netherlands \\ ${ }^{2}$ BioNano Technology, Wageningen University $\&$ Research, Netherlands \\ ${ }^{3}$ Zernike Institute of Advanced Materials, Faculty of Science and Engineering, University of Groningen \\ ${ }^{\dagger}$ Corresponding author: Preeti Sharma, preeti.sharma@wur.nl, JoshuaA. Dijksman, joshua.dijksman@wur.nl
}

\begin{abstract}
Probabilistic fasteners are known to provide strong attachment onto their respective surfaces. Examples are Velcroßand the "3M dual lock" system. However, these systems typically only function using specific counter surfaces and are often destructive to other surfaces such as fabrics. Moreover, the design parameters to optimize their functionality are not obvious. Here, we present a surface patterned with soft micrometric features inspired by the mushroom shape showing a non-destructive mechanical interlocking and thus attachment to fabrics. We provide a scalable experimental approach to prepare these surfaces and quantify the attachment strength with rheometric and video-based analysis. In these "probabilistic fasteners" we find that higher feature densities result in higher attachment force, however, the individual feature strength is higher on a low feature density surface. We interpret our results via a load-sharing principle common in fiber bundle models. Our work provides new handles for tuning the mechanical attachment properties of soft patterned surfaces that can be used in various applications including soft robotics.
\end{abstract}

Keywords: Bioinspiration, mechanical interlocking, load-sharing, 3D printing, soft lithography, attachment devices, soft robotics.

\section{Introduction}

Attachment via mechanical interlocking of three dimensional (3D) protruded features is of importance for many species in Nature [1]. For instance, the arresting system in the head and wings in a dragonfly is found to effectively protect their slim neck; see Figure 1(a) adapted from Jiao et.al. [2]. Also, many other fixation systems in numerous species such as wasps, bees, bugs and beetles have been developed to securely attach their wings to the body while at rest [3]. In the plant world, climbing of the Gallium Aparine plant works via ratchet-like attachment mechanism using micro-macroscopic hooks onto the host plant to seek more sunlight [4-6]. Inspired by such attachment systems found in Nature, smart surfaces consisting micronmillimetric sized attachment features are being developed for many applications such as climbing robots [7] and grippers [8]. The invention of Velcroßwas originally inspired by burdock seeds [9], and the related "3M dual lock" system [10] has been successful for their applications in textile industry and medical field. However, despite providing high attachment forces to their respective opposing surfaces, detachment of these mechanical fasteners leave damage if attached to a fabric.

The essential problem in mechanical attachment is that the interlocking features are rigid, limiting the ability for safe removal without damaging the interlocking features or the fabric itself, also seen in the work from Fiorello et al. [11], where plant-inspired interlocking microstructures having 3D hook shape were tested

\footnotetext{
*These authors contributed equally
} 
(a)

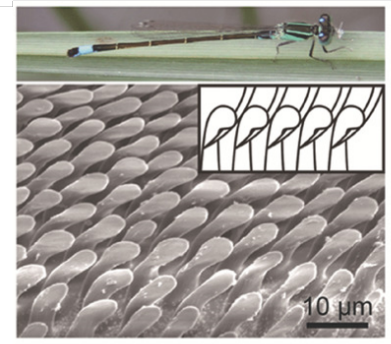

(c)

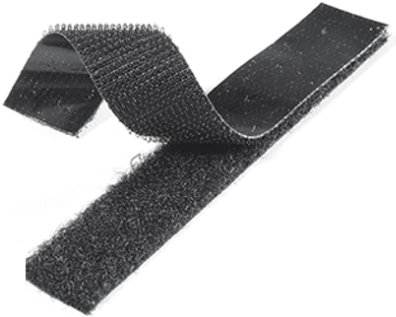

(b)

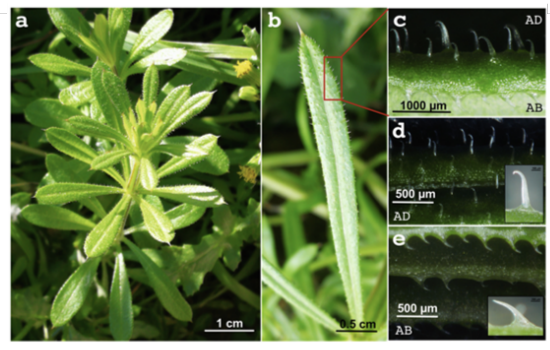

(d)

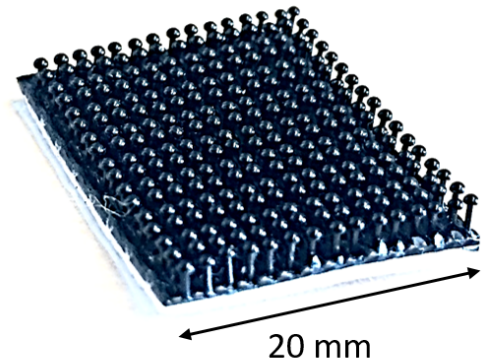

Figure 1: Examples of Natural and man-made interlocking systems (a) Optical image of a dragonfly and micro features responsible for arresting mechanism in head and wings, reproduced from [2]: Copyright 2018, John Wiley and Sons, (b) image of the herbaceous climbing plant G. Aparine and details of leaf and details of interlocking features in zoomed images, reproduced by permission from [14]: Copyright 2018, Springer Nature, (c) a picture of Velcroß) and (d) a picture of the $3 \mathrm{M}$ dual lock taken from camera.

for their attachment on fabric. Another recent study from Fiorello et.al. shows that, if a shear force parallel to the surface was applied, the interlocked features detach without producing damage on different fabrics[7]. Surface directionality can thus resolve the damage issue, yet also limits the applicability of the attachment device. Geometries other than plant-inspired hooks such as pestle, mushroom, and Velcro hooks have also been explored for their attachment to fabrics. It was found that pestle shapes were creating the least damage onto the features and lint of fabric, but with weaker detachment force in normal direction compared to other geometries [2]. It is clear that having features with overhang such as a mushroom shape or Velcro hooks are required to enhance attachment strength normal to the surface, however the stiffness of the features results in partial or permanent damage of the interlocking features or the fabric itself. Having soft patterned surfaces with soft interlocking features would thus be an innovative step towards a new class of damage and residue-free dry adhesives that can actually attach to fabrics of different mesh sizes and potentially even to other surfaces with micro- to macroscopic roughness.

In this work we present a soft surface, functionalized with soft micrometric mushroom-shaped features, providing mechanical interlocking, while leaving no damage on attachment features or the surface it attaches to. We describe a scalable production mechanism by combining simple 3D printing and soft lithography to achieve microscopic patterns in a soft polymer. Subsequently, we show that the surface patterns have the desired mechanical functionality; specifically, the surfaces attach to three different types of fabrics having regular and irregular mesh sizes. The soft features do not damage the fabrics, even though the attachment is based on mechanical interlocking. Apart from assuring safe detachment, we provide insight into the design parameters of the attachment mechanism by probing the interaction between the attachment features. We provide a detailed perspective for tuning the attachment strength of a soft probabilistic fastener via loadsharing rules known from rupture theory in fiber bundle models $[12,13]$. 


\section{Materials and Method}

\subsection{Fabrication of microscopic patterned surfaces}

Conventional lithography techniques are known for being able to create microscopic patterned surfaces in soft elastomers, indeed with high resolution, including complex 3D structure such as mushrooms having a flat disk shape hat [15-18]. Realizing micrometric mushroom features having spherical-like shapes such as "3M dual lock" or a half-spherical hat that is essential for mechanical interlocking has not been possible below a millimeter scale. At present, 3D printers allow an easy and direct realization of complex 3D features such as microfeatures inspired by rose prickles [14], and Salvinia Molesta [19]. We have printed surfaces patterned with 3D mushroom features having spherical hats using a stereolithography 3D printer. The capability of a $3 \mathrm{D}$ printer to print a variety of features other than mushroom as small as $200 \mu \mathrm{m}$ is given in the Supporting Information section 1. A picture of a 3D printed sample having mushroom features can be seen in figure 2 . The samples have been designed in a 3D computer-aided design (CAD) software (SolidWorks 2017), CAD files are attached in the supporting information. The geometrical details of the features in 3D CAD and the 3D printed outcome are illustrated in Table 1. The microfabricated features are in good agreement with the CAD designs for their dimensions and shape.

The cured resin from the 3D printer is a stiff material with Young's modulus of 2.8 GPa [20]. Attachment and subsequent detachment from an opposing surface such as a fabric can break either the small 3D printed features or damage the fabric itself. Therefore, we replicated the 3D printed structure in polydimethylsiloxane elastomer (PDMS) using a double molding process [21-23]. At first, the positive 3D printed structure was replicated as negative in an elastomer ecoflex 0030. Afterward, PDMS Sylgard 184 was cast and cured in the negative ecoflex for making the 3D printed replica, see figure 2 and supporting information section 3 . The Young's Modulus of the PDMS Sylgard was about 1.8 MPa for a mixing ratio of 1:10 and curing temperature $70^{\circ}[24]$.

Table 1: Geometrical details of the mushroom features designed in CAD and 3D printed outcomes shown in figure $2(\mathrm{a}) . H$ is the mushroom stem height, $W$ is the diameter of the stem, $D$ is the periodicity of the features, and $D_{h}$ is the diameter of the mushroom hat (see figure $2(\mathrm{a})$ ).

\begin{tabular}{lllll}
\hline Mushrooms & $H(\mu m)$ & $W(\mu m)$ & $D(\mu m)$ & $D_{h}(\mu m)$ \\
\hline CAD model & 600 & 400 & 1600 & 800 \\
3D printed & $594 \pm 38$ & $400 \pm 15$ & $1600 \pm 2$ & $766 \pm 35$ \\
\hline
\end{tabular}

We found that the alternative method of first 3D printing the negative mold and then casting the PDMS on it does not work for two reasons: printing negative molds having complex 3D features is technically not possible with the printers used, and small features on the PDMS tend to detach while peeling it off the 3D printed mold. Therefore, using double molding is necessary. Ecoflex is equally important, as it is a softer elastomer than PDMS and can be elongated to more than $900 \%$ of its original size, allowing easy demolding of the PDMS. This process results in similar 3D printed features as shown in figure 2(b) with shrinkage of less than $2 \%[25]$.

Another important aspect to take into account is the chemical composition of the different materials and the chemical reaction involved in the process. In fact, 3D printer resins are usually composed of methacrylates, which are reactive with the vinyl-terminated siloxanes present both in the ecoflex and PDMS. This means that if the 3D printed structure is not completely cured, it will react with the uncured ecoflex and it will chemically bond to it, making the peeling off impossible. Similar considerations are relevant when casting PDMS on ecoflex [26]. If the latter is not properly cured, the two silicone based elastomers will react and seal together. We solved those issues using a chemical surface modification of the 3D printed and ecoflex mold. Both surfaces were first activated by a plasma oven and then reacted with perfluorodecyltrichlorosilane (PFOTS) using a chemical vapor deposition (CVD) approach, see supporting information section 2 . In this way the entire surface, both on the $3 \mathrm{D}$ printed positive and the negative ecoflex were made nonreactive 

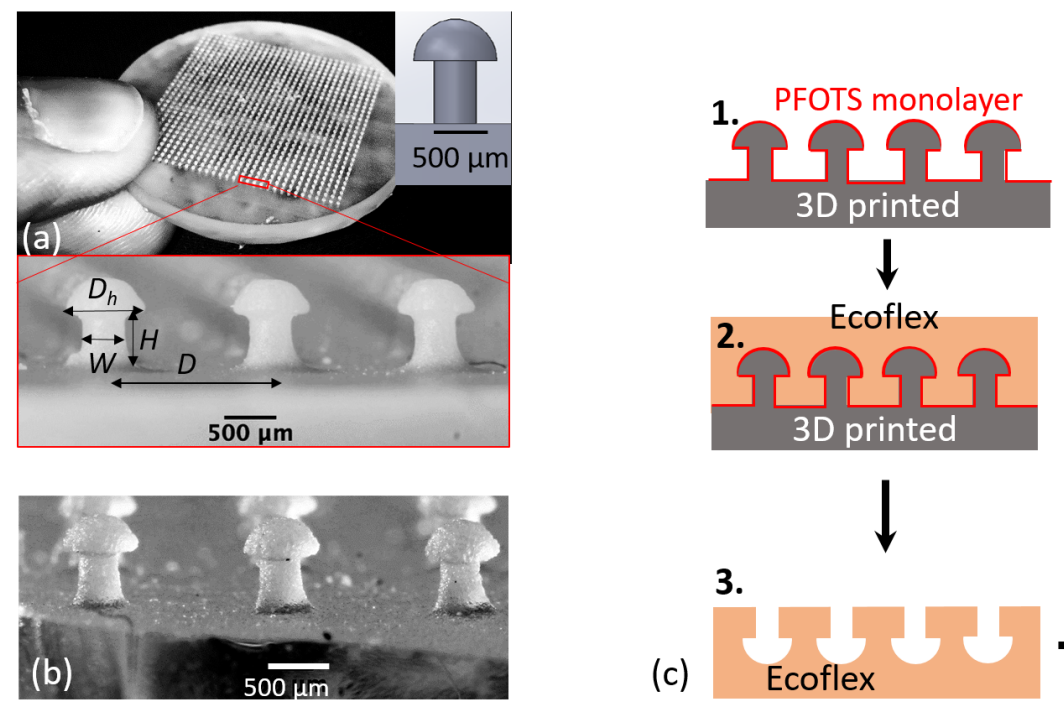

1

3.

(c)
6.

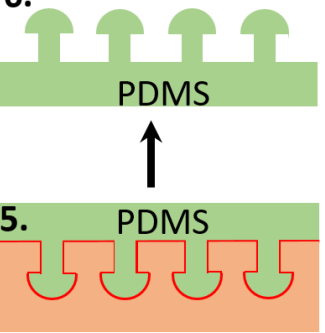

4.

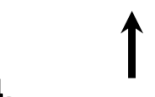

Figure 2: (a) A picture of a 3D printed sample having mushroom features on it and corresponding side view of features in zoomed picture and side view from CAD model in the inset, (b) Microscope picture of the replica of the 3D printer features in PDMS, and (c) schematic illustration of double molding procedure; 1. 3D printed mold 2. molding ecoflex on 3D printed, 3. negative mold in ecoflex 4. monolayer deposition of perfluorodecyltrichlorosilane (PFOTS) by chemical vapor deposition 5. casting PDMS in ecoflex mold and 6. PDMS sample. 


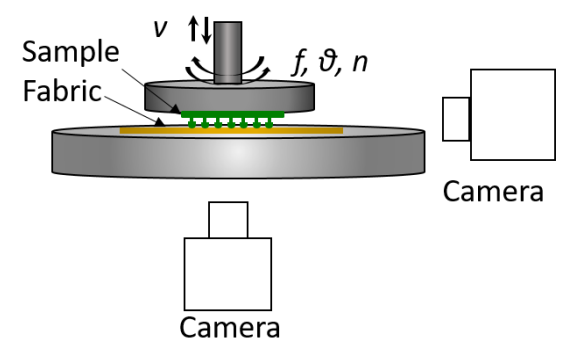

(a)

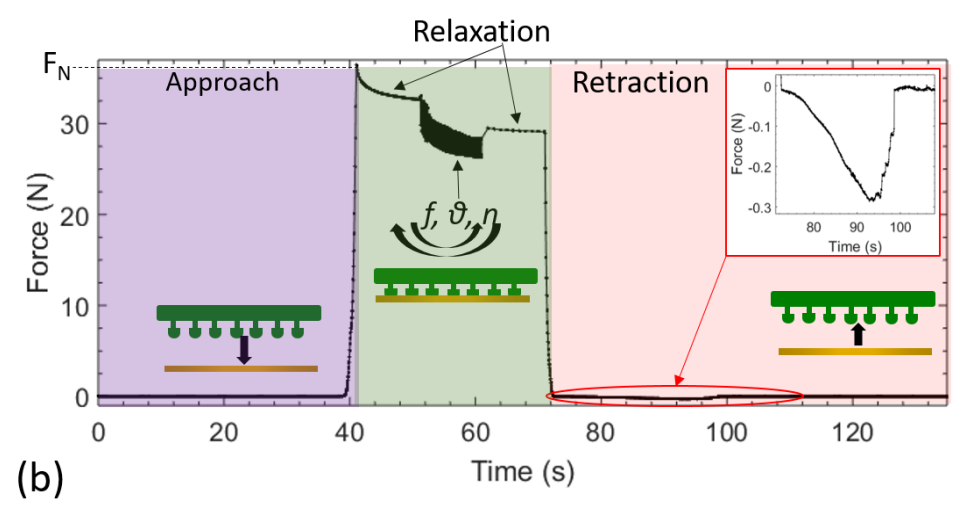

(b)

Figure 3: (a) Schematic of the experimental set-up and (b) typical force curve showing the complete experimental procedure. $v$ is the velocity of the top plate probe during approach and retraction, $f, \theta$ and $n$ are the rotation frequency, angle of oscillations and number of cycles of the top plate respectively.

towards the hydrosilylation curing reaction.

\subsection{Experimental set-up and protocol}

A schematic of the experimental set-up to measure the pull-off force and explore the underlying mechanisms is depicted in figure 3(a). We used a rheometer (Anton Paar MCR 501) in plate-plate configuration to record the force-distance curves and hence to estimate the attachment force. Note that rheometers are more typically known as instruments to measure the viscoelastic properties of liquids; here we use this instrument for attachment force measurements generated by attachment of mushroom features because of their ability to apply an angle of rotation with a desired frequency and number of oscillations along with a known normal force. As such, they mimic the attachment procedure used in qualitative experiments in section 3.1.

A single layer of fabric was fixed at the edges onto a glass substrate with scotch tape, see schematic in Supplementary information section 5, figure 3, which is then fixed at the bottom plate of the rheometer using UV curing glue (Norland product). A sample with mushroom features is fixed onto a glass substrate using plasma oxidation which is afterward fixed onto a top plate of the rheometer via a drop of UV curing glue. The glue is hardened while the sample is in contact with the counter surface at the bottom plate of the rheometer so that both of the contact surfaces are aligned with one another. Two cameras are used to record the attachment mechanism: one camera is placed under the transparent bottom plate of the rheometer and another one is observing the attachment dynamics from the side.

Four samples of identical size with varying feature density, i.e. $25,39,69$, and 83 mushroom $/ \mathrm{cm}^{2}$, have been used to test the attachment performance on a Nylon based synthetic fabric, abbreviated as SIF in this work. The protocol that has been used to measure attachment force with the rheometer consists of the steps described here and shown in figure 3(b). First, the sample makes an approach towards the fabric sample with a velocity $v=500 \mu \mathrm{m}-\mathrm{s}^{-1}$ to reach a normal force $F_{N}$. After a relaxation of 10 seconds, the top plate oscillates at an angular frequency $f$ along with a given rotation angle $\theta$ for the number of cycles $n$. We then let the combined surfaces relax for 10 seconds while keeping the position of the top plate fixed. Finally, the top plate is retracted with the same velocity as in the approach step. In all experiments, the angle used for oscillatory motion $\theta$ is fixed to $10^{\circ}$ as to best approximate the qualitative experiments demonstrated in section 3.1. 


\section{$3 \quad$ Results and Discussions}

\subsection{Attachment to fabrics}

At first, patterned surfaces were tested qualitatively for their attachment on three types of fabrics (Figure 4): (1) Nylon based synthetic fabric commonly known as pantyhose, which is highly stretchable in nature and has an irregular mesh size in the unstretched state. We abbreviate it as SIF (stretchable irregular fabric) in this work; (2) Non-stretchable synthetic and cotton mixed fabric with a fixed mesh size of $450 \times 750 \mu \mathrm{m}$ called nSRF1 (non-stretchable regular fabric 1); and (3) A non-stretchable cotton fabric with a fixed mesh size of $250 \times 300 \mu \mathrm{m}$ known as cheese-straining cloth and abbreviated as nSRF2. 3D printed samples were pressed manually against the fabric which results in no attachment of features. To obtain mechanical interlocking of shapes with overhang in a substrate with openings, we posit that a small angular rotation with a normal pressure is essential as it allows deformation in the fabric to let the features interlock. In the case of PDMS sample, the mushroom features also bend and the movement induces the mushroom heads to get stuck into the fabric opening, see supporting information section 6 and video 1 for a visual representation.
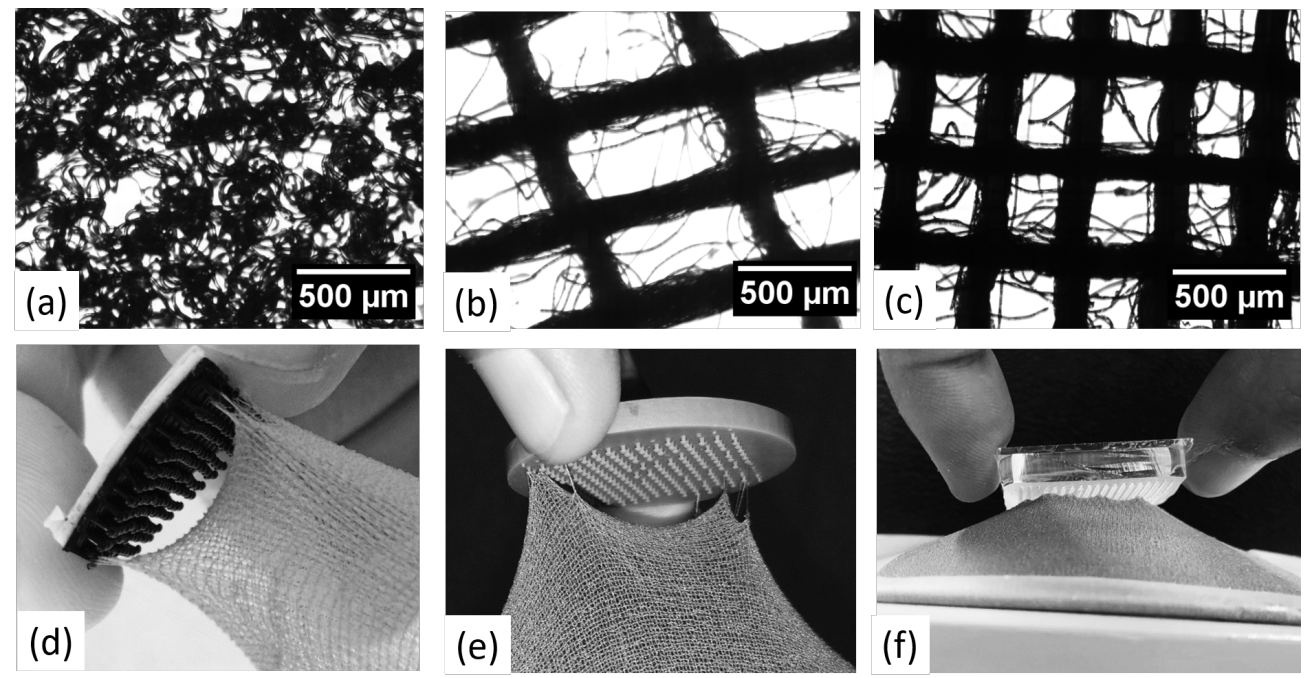

Figure 4: Microscopic pictures of the fabrics: (a) Stretchable irregular fabric (SIF); (b) Non-stretchable regular fabric 1 (nSRF1); (c) Non-stretchable regular fabric 2 (nSRF2). Images were taken during qualitative attachment experiments of (d) 3M dual lock sample on SIF; (e) 3D printed sample having mushroom structures on SIF and, (f) PDMS sample with mushroom features onto SIF.

At first, the attachment of 3D printed sample and a piece of 3M dual lock mechanical fastener has been tested. Both samples tend to adhere to the SIF and nSRF1 fabric but at the expense of damage on the fabric as shown in figure 4(d) and (e). Also, the rupture of a few mushroom features was observed in the 3D printed sample; see figure 4(e). No damage was observed for the nSRF2 fabric as no mechanical interlocking was observed since the size of mushroom features being significantly larger than the textile mesh size. However, a sample printed with smaller mushroom features $(\mathrm{W}=200 \mu \mathrm{m}, \mathrm{H}=400 \mu \mathrm{m}, \mathrm{R}=200 \mu \mathrm{m})$ shows evidence of attachment to the nSRF2 fabric, but again at the expense of permanent damage on either sample or fabric. The soft mushroom sample also adheres to all fabrics. No damage was observed on fabrics or the PDMS sample itself, see figure 2 in Supplementary information section 4 . For a complete overview of attachment performance, see Table 2 . 
Table 2: Qualitative results from the three samples on three types of textiles. $\checkmark$ denotes attachment on fabric.

\begin{tabular}{llll}
\hline Textiles & 3M dual lock & 3D printed sample & PDMS sample \\
\hline SIF & $\checkmark$, damage on fabric & $\checkmark$, damage on fabric and sample & $\checkmark$, no damage \\
nSRF1 & $\checkmark$, damage on fabric & $\checkmark$, damage on fabric and sample & $\checkmark$, no damage \\
nSRF2 & no attachment & $\checkmark$, damage on fabric and sample & $\checkmark$, no damage \\
\hline
\end{tabular}

\subsection{Quantification of attachment}

Figure 5(a) shows the force-displacement curves of a sample having a feature density of 39 features-cm $^{-2}$ on $^{-}$ SIF fabric at $F_{N}=5 N, f=5 \mathrm{~Hz}$. The red curve is for $n=0$ and black for $n=50$. See supporting information section 6 , video 2 to visualize the dynamics as recorded during the measurement that produced the black curve. From this experiment, it is clear that pressing is not sufficient to get the mushroom features interlocked into the fabric, hence, no attachment force was observed; see red curve (zero cycles). In both experiments, the retraction curve is separated from the approach curve, presumably because of some viscoelastic mechanism playing a role, perhaps due to the sliding of both the PDMS mushroom features and fabric on the glass plate. During retraction, pull-off events of varying sizes can be seen because of the subsequent detachment of a number of mushroom-fabric pairs. The peak force from this curve is used to characterize the attachment force.

First, we have optimized the oscillation frequency and number of cycles at $5 \mathrm{~N}$ normal force. We so minimize the variables for a systematic study of the attachment dynamics. The attachment force initially increases linearly with the number of cycles, after which it reaches a plateau. The transition is roughly around 20 cycles, irrespective of the frequency of oscillations. The saturation suggests that there is saturation in the number of attached features to the fabric. See figure 5(b). Clearly, the adhesive elements are interlocking on the surface, however, a visual inspection of the micrometric features is required for a better understanding of interlocking dynamics. We captured videos simultaneously with attachment experiments and detailed image analysis is given in Supporting Information section 3. The result is shown in figure 5(c); on the left, the outcome of the image analysis for a sample having feature density 69 features- $\mathrm{cm}^{-2}$ when preloaded from $10-35 \mathrm{~N}$ and, on the right, the fraction of the interlocked features to the fabric during oscillation phase is plotted against normal force at a constant oscillation frequency of $5 \mathrm{~Hz}$ and 50 cycles at the right. Note that the normal forces required to achieve interlocking are substantially higher than the attachment forces obtained. We attribute this to the fact that the mushrooms have to physically penetrate the fabric, making this mechanism strain dependent. The soft mushroom features compress and bend easily and in their current design need a relatively large compressive load to make their way through the fabric. Shorter mushroom stems and other geometric design features can thus be used to reduce the normal forces required.

At low normal force, a certain number of mushroom-fabric pairs are formed out of available mushroom features on the sample. Indeed, a few distinctive regions of paired mushrooms can be seen, see $5(\mathrm{c})$, that are certainly responsible for the pull-off events seen in force-distance curves, see figure 5 (a). As the normal load is increased, an expansion or creation of new paired regions occurs due to the interlocking of more features into the fabric. At a certain normal force, regions of paired mushrooms merge, and afterward, the linear increment in paired features starts to slow down reaching a saturation regime, as most of the mushrooms are already interlocked. See figure $5(\mathrm{~d})$ where the fraction of paired mushrooms vs normal force is shown. The available features are more abundant than the actually interlocked features, as is clear from figure 5(c). Not all features interlock in the saturation regime as mushroom penetration is sensitive to the angular rotation. The mushroom features in the middle of the sample perceive a lower activation strain and rarely get interlocked to the fabric. Also, we can see in figure $5(\mathrm{~d})$ that interlocking of the soft features is more obvious for the highest feature density; the presence of neighboring mushrooms stimulates mushroom penetration.

The interlocking strength of the individual features is plotted in figure $6(\mathrm{a})$. To clarify trends, the 

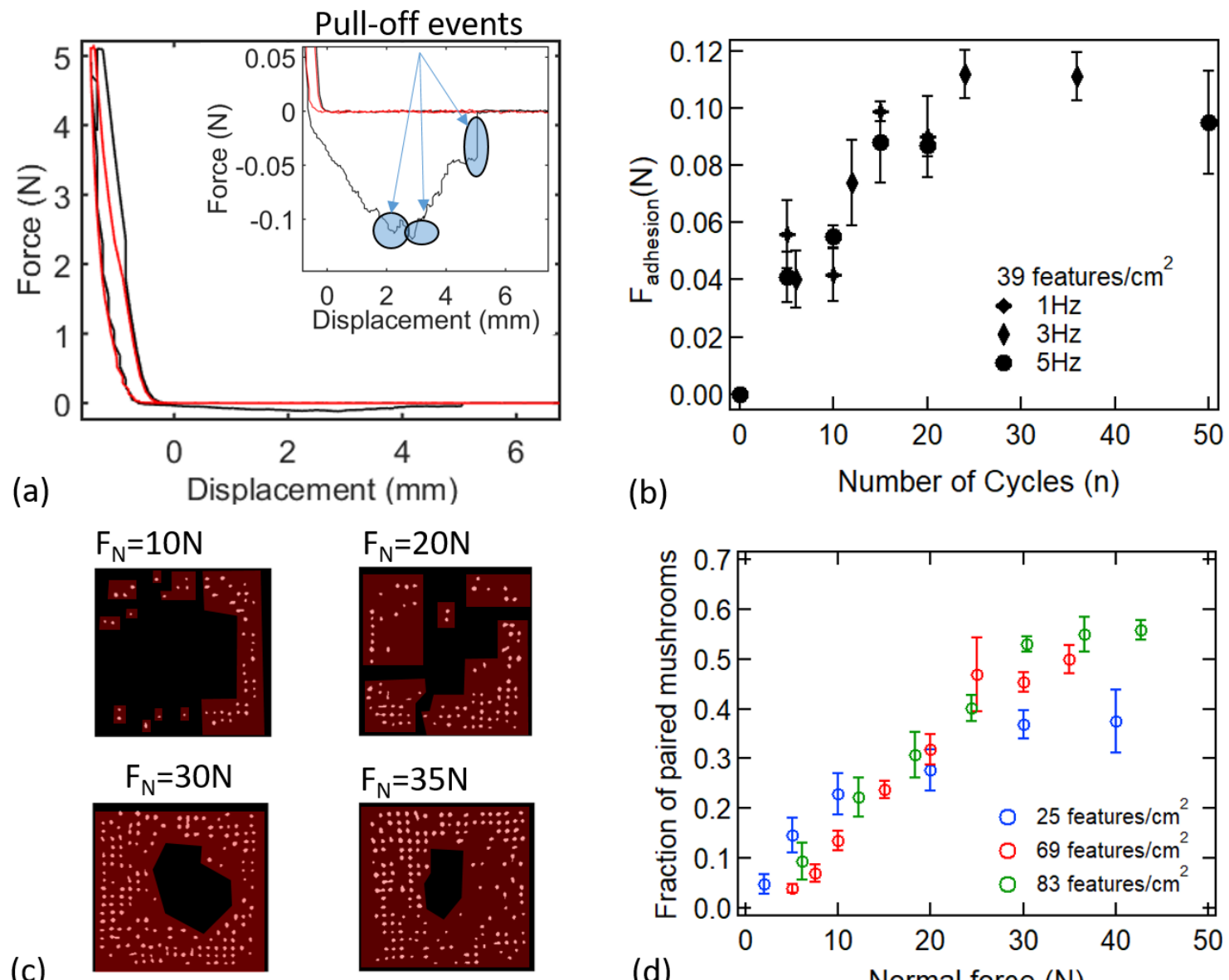

(c)

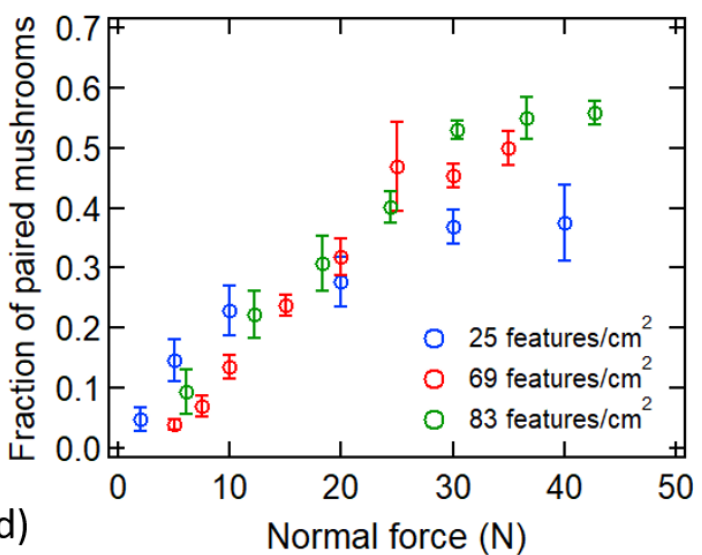

Figure 5: (a) Force-displacement curves of mushroom sample having 39 features-cm ${ }^{-2}$ on SIF fabric, $v=500$ $\mu \mathrm{m} / \mathrm{s}, f=5 \mathrm{~Hz}, \theta=10^{\circ}, n=0$ for red and $n=50$ for black, inset is showing the zoom of the curve during retraction. (b) Attachment force vs number of cycles at frequencies $1 \mathrm{~Hz}, 2 \mathrm{~Hz}$ and $5 \mathrm{~Hz}$. Normal force was $5 \mathrm{~N}$. Interfacial dynamics of features: (c) result of image analysis of a sample 69 features- $\mathrm{cm}^{-2}$ preloaded at four different normal forces: dots are paired mushrooms to the fabric in black background and the regions of paired mushrooms are represented by the red shade and (d) fraction of interlocked mushrooms as a function of the normal force, $f=5 \mathrm{~Hz}, \theta=10^{\circ}, n=50$ 

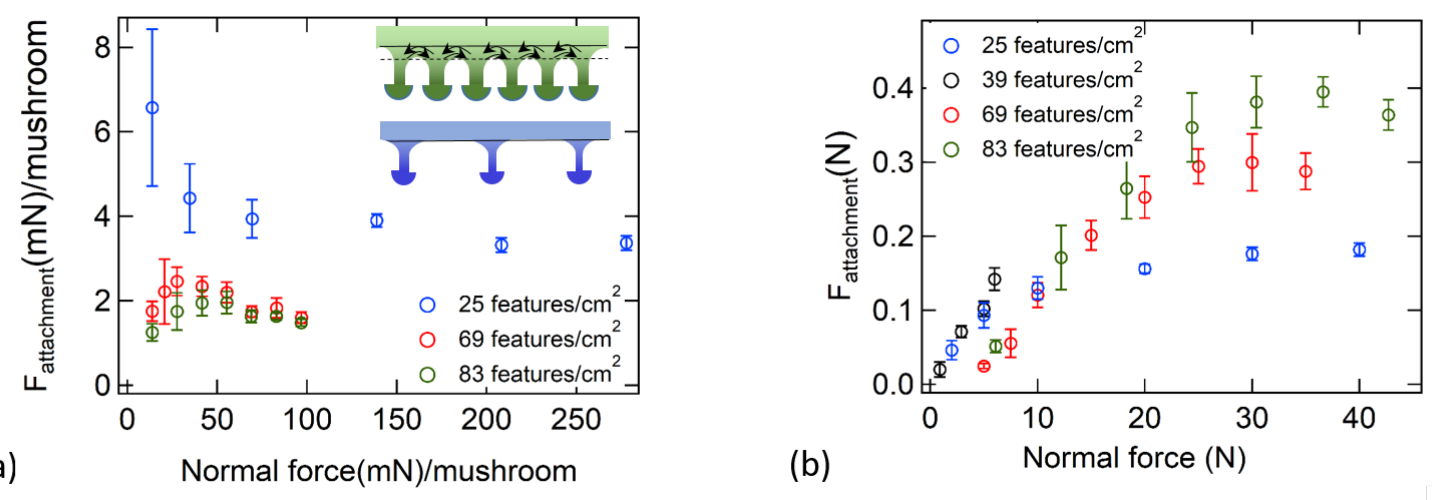

Figure 6: (a) The interlocking force of individual paired features vs preload per unit feature, inset is a schematic illustration of increasing inter-pillar communication with decreasing pillar distances via the substrate deformation. and (b) Attachment force vs normal force for all samples with varying feature density, $f=5 \mathrm{~Hz}, \theta=10^{\circ}, n=50$.

attachment force was normalized by the total number of paired features and is plotted against normal force per unit available features on the sample. We found that the interlocking force is higher in the case of a less dense sample, which means that the individual soft mushroom features are more strongly attached to the fabric. We attribute this to large inter-pillar distances, which result in less inter-pillar communication. This is schematically explained in the inset of figure 6(a), where an array of mushroom pillars is shown in the case of small and large interpillar distances. As a result of an exerted force on the pillars during detachment, it is to be expected that the backing layer deforms as well [27]. Depending on the inter-pillar distance this deformation in the elastic matrix can couple strongly to other pillars. As a result, the lost load during detachment of an individual pillar is distributed locally or globally on the remaining attached features. From rupture studies in fiber bundle models, this so-called "local load sharing" is known to lead to catastrophic detachment of the entire paired region $[13,28]$. Such effects of load sharing have been exploited experimentally in gecko-inspired elastomeric microfibrillar adhesives, by Song et.al [29], where the maximum adhesion force was found to be 14 times larger than the adhering membrane in local load-sharing case. We suggest that this mechanism is present in the mechanical interlocking based elastomeric adhesive as well, attributing the lower value of the individual pillar strength for samples having small inter-pillar distances i.e. for with higher feature density. When the inter-pillar distance increases, the mushroom-fabric pair breaking follows most likely "global load-sharing" that results in a higher value of individual pillar strength during detachment as can be seen in the case of the sample having a lower feature density of 25 features $/ \mathrm{cm}^{2}$ in figure $5(\mathrm{~d})$.

Global load sharing mechanics also induces another counter-intuitive effect. The total adhesive strength of various samples with varying density of the features is depicted in figure 6(b). The attachment force increases linearly with increasing the normal force in agreement with figure $5(\mathrm{c})$. At a certain normal force, saturation is observed, followed by a slight decrease in force, even though there is an increase in the number of interlocking mushrooms features as recorded in figure 5(c). This happens due to the load-sharing effects explained in the previous paragraph: at a certain preload, a merging of regions of paired mushrooms occurs, which induces elastic coupling and hence a decrease in the detachment strength of individual interlocked mushroom with the normal force, see figure 6(a). Despite the effectiveness of global load sharing for low asperity density surfaces, the total adhesive strength of a low asperity density surface is still lower for our particular mushroom asperity design. Having a higher feature density is favorable for increasing the overall attachment strength of soft mechanical adhesives simply due to the number of extra features on the surface. The dependence of the attachment strength of a patterned surface with mechanical interlocking features on 
the normal force has been reported in earlier works as well. In a study on plant-inspired microhooks, Fiorello et.al. [7] observed an increase in the detachment strength while increasing preloading force presumably more features were engaged in the interlocking mechanism. In another study of beetle-inspired wing-locking devices, Pang et.al. observed higher detachment force due to higher overlapping of the micro-nanometric pillar [30]. However, to the best of our knowledge, our work is the first visual demonstration of the microscopic details of interlocking features. Also, our analysis based on load-sharing shows nontrivial scaling rules in the design of soft probabilistic fasteners. In our work, taking the fact that the highest density of the mushroom features results in the lowest attachment force per mushroom due to large elastic coupling, the maximum detachment force of soft mushrooms is $64 \mathrm{mN} / \mathrm{cm}^{2}$. This peak force which is undoubtedly smaller than the work reported by Fiorello at.al., where the attachment of plant-inspired hooks onto polyester fabric were tested [7], and by Jiao at.al., where the pestle-loop mechanical interlocker were tested onto a knitwear fabric [2], also see table 2 of Supplemental Information. This is partly due to the difference in texture and mechanical properties of the studied fabrics and partly due to the stiffness of the interlocking features. The interlocking features in our work are elastic and soft: the Young's modulus is approximately three orders of magnitude smaller than the literature mentioned above. Even being less stiff the features are able to get interlock to the fabric and attributing significant attachment force without damaging the interlocking features or the fabric.

To show the adaptability of the attachment features towards a variety of fabrics with varying mesh sizes, we quantified the attachment force of one of the samples (feature density of 39 features- $\mathrm{cm}^{-2}$ ) onto three different types of the fabric described in table 2. The results are tabulated in table 3 for their attachment forces. The force in the case of stretchable fabric (SIF) is found to be smaller than in the non-stretchable regular fabric (nSRF1), even though the mesh size was larger in the case of nSRF1 fabric. The difference here

Table 3: Summary of the measured attachment forces and related mechanisms on three types of fabrics. The error in the measured force comes from the standard deviation of three consecutive experiments.

\begin{tabular}{|c|c|c|}
\hline Fabrics & Attachment force $(\mathrm{mN})$ & Mechanism \\
\hline SIF & $142 \pm 15$ & mechanical interlocking of mushroom features \\
\hline nSRF1 & $167 \pm 29$ & $\begin{array}{l}\text { mechanical interlocking of mushroom feature, lint en- } \\
\text { tanglement on mushroom features }\end{array}$ \\
\hline nSRF2 & $60 \pm 30$ & lint entanglement on mushroom features \\
\hline
\end{tabular}

is probably due to the nature of the nSRF1 fabric. Along with the mechanical interlocking of features, the nSRF1 surface allows for mechanical entanglement of some lint of the fabric around the mushroom features. This was observed directly; see video 3 in supporting information section 5 . The origin of this entanglement was the friction between the PDMS surface and fabric that generates lint. In the case of non-stretchable regular fabric (nSRF2), no mechanical interlocking of mushroom features into the mesh of fabric was observed due to the feature size being larger than the mesh size. Here the attachment force measured comes entirely from the entanglement of the lint of fabric developed due to friction between the features and fabric; see supporting information section 6 , video 4 .

\section{Conclusion}

We have developed soft surfaces patterned with 3D soft mushroom features. We showed how such mushroom shape features can create attachment when paired with fabrics without damaging themselves or the fabric. The attachment of mushrooms is based on interlocking; we demonstrated this by systematically changing the parameters of the custom-designed attachment application protocol. We demonstrated the effect of the feature density on the attachment strength and find that the feature distance controls detachment dynamics in a way reminiscent of fiber bundle model dynamics.

As future perspectives, we highlight that an active mechanism to minimise and/or control the elastic 
coupling between features can further actively enhance the adhesive strength of soft mechanical adhesives. The stiffness of the mushroom features or the backing layer or substrate might play a role and both can be varied in a passive or an active way [31]. Note that the stiffness of the PDMS polymer can be controlled passively by tuning the ratio of the curing agent mixed in the PDMS during the preparation [32]. Also, post-curing the PDMS samples at higher temperatures affects the elastic modulus [24, 33]. Once the scaling rules for attachment force depending on the substrate stiffness are clear and actively controlled, some other design parameters such as the shape or design of the individual interlocking feature itself can be adjusted for further enhancement of the attachment strength. For instance, varying the stem length or diameter of the mushroom shape, or using an arrow-shaped hat instead of a half-spherical mushroom hat are just some of the possibilities that can be explored in the future. Our study so provides a clear route towards a systematic design of soft mechanical interlocking based fasteners.

\section{Acknowledgements}

Funding from the 4TU Federation through the program "Soft Robotics" with grant number 4TU-UIT-335 is gratefully acknowledged.

\section{Data availability statement}

The data that support the findings of this study are available from the corresponding author upon reasonable request.

\section{References}

[1] C. W. Smith, S. N. Gorb, and V. L. Popov, Philosophical Transactions of the Royal Society of London. Series A: Mathematical, Physical and Engineering Sciences 360, 211 (2002).

[2] J. Jiao, F. Zhang, T. Jiao, Z. Gu, and S. Wang, Advanced Science 5, 1700787 (2018).

[3] S. N. Gorb, International Journal of Insect Morphology and Embryology 27, 205 (1998).

[4] K. J. Niklas, Current Biology 21, R199 (2011).

[5] G. Bauer, M.-C. Klein, S. N. Gorb, T. Speck, D. Voigt, and F. Gallenmüller, Proceedings of the Royal Society B: Biological Sciences 278, 2233 (2011).

[6] J. Burris, S. Lenaghan, and C. Stewart, Plant Cell Reports 37, 10.1007/s00299-017-2240-y (2017).

[7] I. Fiorello, O. Tricinci, G. A. Naselli, A. Mondini, C. Filippeschi, F. Tramacere, A. K. Mishra, and B. Mazzolai, Advanced Functional Materials 30, 2003380 (2020).

[8] T. Zhang, T. Liang, X. Yue, and D. Sameoto, in 2019 2nd ieee international conference on soft robotics (robosoft) (IEEE, 2019), pp. 120-125.

[9] G. d. Mestral, Welvet type fabric and method of producing same, 1955.

[10] W. L. Melbye, N. Susan K., L. E. Wood, M. D. Lindseth, and D. A. Bychinski, Mushroom-type hook strip for a mechanical fastener, 2003.

[11] I. Fiorello, O. Tricinci, A. K. Mishra, F. Tramacere, C. Filippeschi, and B. Mazzolai, in Biomimetic and biohybrid systems, edited by V. Vouloutsi, J. Halloy, A. Mura, M. Mangan, N. Lepora, T. J. Prescott, and P. F. Verschure (2018), pp. 168-178.

[12] R. Long, C.-Y. Hui, S. Kim, and M. Sitti, Journal of Applied Physics 104, 044301 (2008).

[13] Y. Mulla, G. Oliveri, J. T. Overvelde, and G. H. Koenderink, Physical Review Letters 120, 10.1103/ physrevlett.120.268002 (2018). 
[14] I. Fiorello, F. Meder, O. Tricinci, C. Filippeschi, and B. Mazzolai, "Rose-inspired micro-device with variable stiffness for remotely controlled release of objects in robotics", in Biomimetic and biohybrid systems (Springer, Cham, 2019), pp. 122-133.

[15] L. Heepe and S. Gorb, Annual Review of Materials Research 44, 10.1146/annurev-matsci-062910$100458(2014)$.

[16] Y. Wang, H. Hu, J. Shao, and Y. Ding, ACS Applied Materials \& Interfaces 6, PMID: 24527733, 2213 (2014).

[17] S. C. Fischer, O. Levy, E. Kroner, R. Hensel, J. M. Karp, and E. Arzt, Journal of the Mechanical Behavior of Biomedical Materials 61, 87 (2016).

[18] M. Seong, H.-H. Park, I. Hwang, and H. E. Jeong, Coatings 9, 48 (2019).

[19] O. Tricinci, T. Terencio, B. Mazzolai, N. M. Pugno, F. Greco, and V. Mattoli, ACS Applied Materials \& Interfaces 7, PMID: 26558410, 25560 (2015).

[20] Materials for high-resolution rapid prototyping, Rev. 2, FORMLABS (Apr. 2016).

[21] H. G. Andrews and J. P. S. Badyal, Journal of Adhesion Science and Technology 28, 1243 (2014).

[22] D. Chandra and S. Yang, Accounts of Chemical Research 43, 1080 (2010).

[23] A. Iuliano, E. van der Wal, C. W. B. Ruijmbeek, S. L. M. in 't Groen, W. W. M. P. Pijnappel, J. C. de Greef, and V. Saggiomo, Advanced Materials Technologies 5, 2000344 (2020).

[24] I. D. Johnston, D. K. McCluskey, C. K. L. Tan, and M. C. Tracey, Journal of Micromechanics and Microengineering 24, 035017 (2014).

[25] M. Madsen, N. Feidenhans'l, P.-E. Hansen, J. Garnaes, and K. Dirscherl, Journal of Micromechanics and Microengineering 24, 127002 (2014).

[26] A. Iuliano, E. van der Wal, C. W. B. Ruijmbeek, S. L. M. in 't Groen, W. W. M. P. Pijnappel, J. C. de Greef, and V. Saggiomo, Advanced Materials Technologies 5, 2000344 (2020).

[27] I. N. Sneddon, International Journal of Engineering Science 3, 47 (1965).

[28] "Local and intermediate load sharing", in The fiber bundle model, edited by A. Hansen, P. C. Hemmer, and S. Pradhan (John Wiley \& Sons, Ltd, 2015) Chap. 4, pp. 63-114.

[29] S. Song, D.-M. Drotlef, C. Majidi, and M. Sitti, Proceedings of the National Academy of Sciences 114, E4344 (2017).

[30] C. Pang, D. Kang, T.-i. Kim, and K.-Y. Suh, Langmuir 28, 2181 (2012).

[31] M. Tatari, A. Mohammadi Nasab, K. T. Turner, and W. Shan, Advanced Materials Interfaces 5, 1800321 (2018).

[32] J. Park, S. Yoo, E. Lee, D. Lee, J. Kim, and S. Lee, English, Biochip Journal 4, 230 (2010).

[33] M. Kim, B.-U. Moon, and C. H. Hidrovo, Journal of Micromechanics and Microengineering 23, 095024 (2013). 